\title{
Multislice HAADF STEM Image Simulation of Complex Oxidation Catalyst "M1".
}

\author{
Douglas A. Blom ${ }^{1}$
}

1. NanoCenter and College of Engineering and Computing, University of South Carolina, Columbia, SC, USA

Annual worldwide acrylonitrile production is nearly $1 \mathrm{~kg} /$ person [1]. Currently, production is via the "SOHIO process" originally developed in the 1950s [2]. Propylene precursor is reacted over a multiphase catalyst in the presence of ammonia. For both economic and energy use reasons, there is a strong desire to produce acrylonitrile using propane as the starting material. This requires the development of a new catalyst. The most promising catalyst for the direct ammoxidation of propane to acrylonitrile is a quatenary Mo-V-Nb-Te oxide phase originally reported by the Mitsubishi Chemical Co. and therefore typically referred in the literature as "M1". The crystal structure was originally solved with the combined Rietveld refinement of synchrotron X-ray and neutron powder diffraction data [3]. More recently, the model was improved due to input from HAADF STEM observations [4]. Figure 1 is the structural model of the cations in this phase in a $\{001\}$ orientation. The unit cell consists of a series of pentagonal, hexagonal and heptagonal rings of metal-oxygen octahedra. The center of the pentagonal ring is populated by the $\mathrm{Nb}$ in the crystal. Te and oxygen chains are present in both the hexagonal and heptagonal channels. The other 10 crystallographically distinct cation sites are populated by various mixtures of Mo and V.

Previously, we have published the results of multi-slice ADF STEM image simulations on this material [5]. The simulations were performed for the older structural model from [3]. In addition, the virtual crystal approximation (VCA) was used in the published simulations. The projected potential of the cation sites with partial substitution of Mo and V in the VCA is the weighted average of the potentials of Mo and V. For STEM, the ADF signal is sensitive to not only the average mass of the cations, but also the location of the cations along the beam propagation direction [6]. In this work, we report the results of multi-slice ADF STEM image simulations for the improved structural model [4] without the VCA.

Frozen phonon simulations using the multislice code of Kirkland [7] were performed. An accelerating potential of $200 \mathrm{kV}$, convergence semi-angle of $17.3 \mathrm{mrad}$, Cs $3 \mu \mathrm{m}$, C5 0, defocus $2 \mathrm{~nm}$ were the common microscope parameters. The ADF detector in the simulation spanned 100-425 mrad. 64 phonon passes were used. The simulations were carried out for an area slightly larger than $1 / 4$ of the unit cell which contained all the cation sites for the structure (see Fig. 1). 30 unit cells along the c axis $(12 \mathrm{~nm})$ was the simulated thickness. The atomic coordinates, thermal parameters, and occupancy were taken from [4]. Several simulations with the same occupancy of Mo and V but different configurations along the beam propagation direction were run. A random solid solution was assumed. Figure 2 is a graphical representation of the cation distribution along the beam direction was for 13 independent cation sites for a single configuration. The intensity is equal to the atomic number of the cation for all 30 possible locations The last two columns are the Te containing sites that are either empty or Te. Integrated intensities for the ADF simulation of the S1 site $(30 \% \mathrm{~V})$ is shown in Fig. 3 for 7 different configurations. The image intensity varies by $9 \%$ relative to the mean depending on the cation distribution [8].

References: 
[1] http://www.prweb.com/releases/2014/04/prweb11740156.htm retrieved 02/19/2015.

[2] http://www.acs.org/content/acs/en/education/whatischemistry/landmarks/acrylonitrile.html retrieved $02 / 19 / 2015$.

[3] P. DeSanto et al., Z Kristallogr. 219 (2004), p.152.

[4] X. Li et al., Top Catal. 54 (2011), p.614.

[5] D. A. Blom, Ultramicroscopy, 112 (2012), p.69.

[6] E. Carlino and V. Grillo, Phys Rev B, 71 (2005), p. 235.

[7] E.J. Kirkland, Advanced Computing in Electron Microscopy, $2^{\text {nd }}$ ed. Springer, New York, 2010..

[8] The author acknowledges funding from the National Academies Keck Future Initiative. This work used the Extreme Science and Engineering Discovery Environment (XSEDE), which is supported by National Science Foundation grant number ACI-1053575

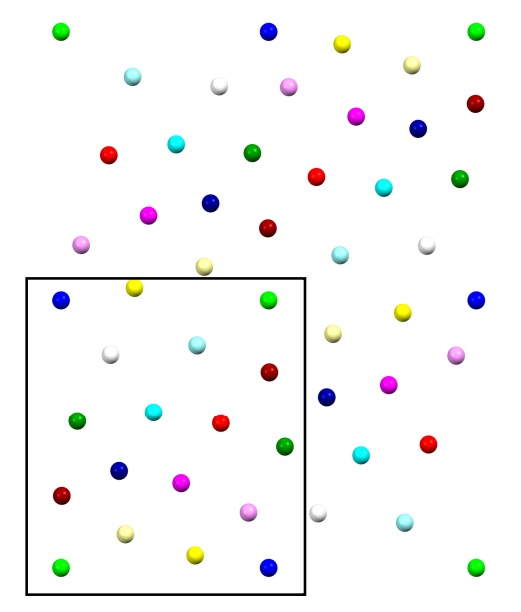

Figure 1. Cation positions in the 001 projection of the Mo-V-Nb-Te oxide M1 catalyst. Color correspond to crystallographically distinct atom positions. The box shows the area of the image simulations.

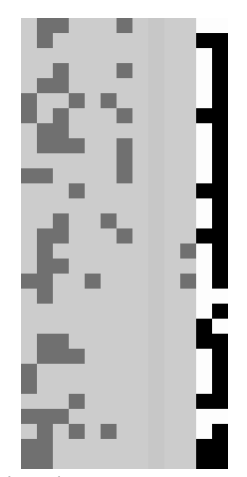

Figure 2. Graphical representation of the cation distribution along the electron propagation direction. Each column corresponds to one of the unique cation sites in the unit cell. The intensity is equal to the atomic number of the cation.

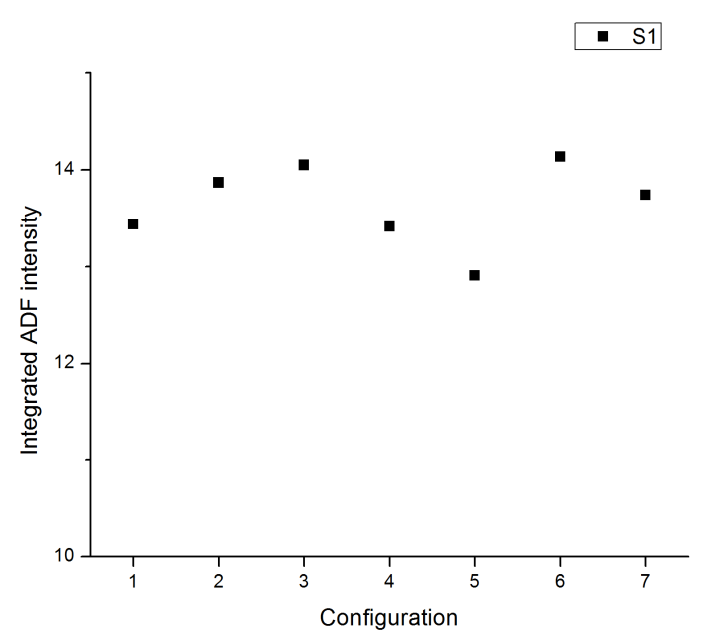

Figure 3. Integrated ADF intensity for cation site 1 for 7 different orderings of $\mathrm{V}$ and $\mathrm{Mo}$ along the beam direction. The composition is constant, but the ADF intensity varies by $9 \%$. 